\title{
CREATION OF A FRAMEWORK OF DESIGN TOOL CHARACTERISTICS TO SUPPORT EVALUATION AND SELECTION OF VISUALISATION TOOLS
}

\author{
Zhang, Wenwen; Ranscombe, Charlie; Radcliffe, David; Jackson, Simon \\ Swinburne University of Technology
}

\begin{abstract}
In Industrial Design, new design visualisation tools are emerging offering significant benefits to the designers. However, studies show alongside some benefits, new tools can also inhibit designers' creativity or cause time inefficiency if used in the wrong context. Thus, understanding which tools to use and when during the design process is increasingly necessary to ensure the best use of resources in design practice. Existing research on understanding the performance of design tools and the resulting frameworks for comparing tools are either specific to certain tools or highly generalised making evaluation across different design tools challenging. As such, this paper reports the creation of a more comprehensive framework of design tool characteristics to facilitate a better understanding of design tools and their uses. Demonstration of application of the framework is also given in the form of a case study on the use of Digital Sketching and its comparable tools with four practising designers. In conclusion, we show how the Design Tool Characteristics (DTCs) framework is an effective way to understand design tools, with further implications for design tool development.
\end{abstract}

Keywords: Evaluation, Design Tool Characteristics, Visualisation, Decision making, Case study

\section{Contact:}

Zhang, Wenwen

Swinburne University of Technology

School of Design

Australia

wenwenzhang@swin.edu.au

Cite this article: Zhang, W., Ranscombe, C., Radcliffe, D., Jackson, S. (2019) 'Creation of a Framework of Design Tool Characteristics to Support Evaluation and Selection of Visualisation Tools', in Proceedings of the 22nd International Conference on Engineering Design (ICED19), Delft, The Netherlands, 5-8 August 2019. DOI:10.1017/dsi.2019.117 


\section{INTRODUCTION}

Rapidly emerging digital visualisation tools offer significant benefits to industrial designers regarding time efficiency and presenting photo-realistic design representations. Such tools include digital sketching, 3D CAD modelling, 3D printing, virtual reality devices, etc. Lutters et al. (2014) report that the selection of tools during design activity as mostly relying on the designers' expertise and creativity. However, research shows that new visualisation tools can inhibit designers' creativity and/or be time inefficient if used in the wrong context (Robertson and Radcliffe, 2009). Thus, a concern for designers is making decisions about which tools to use, and when, during the design process, to optimise the time efficiency and achieve effective design outcomes. Unfortunately, the choices of what tools to use, and when, can't be made only from knowing which tools are available. The more we understand the design tools and their alternatives, the greater chance we have to figure out their most suitable context and the better use of them in design practice.

As Lutters et al. (2014)state, characteristics of techniques and tools are more efficient, credible and useful in capturing the essence of tools than collecting endless lists of existing tools. In other words, the key to effectively understanding and evaluating various design tools is to identify comparable characteristics. Existing research into evaluating the performance of design visualisation tools and resulting frameworks are either specific to certain tools or generalised at a level of designer behaviour (Self, 2011; Pei et al., 2011). As such, a more comprehensive framework of design tool characteristics for understanding design tools and conducting design tool comparisons is built.

This paper presents the creation of this more comprehensive Design Tool Characteristics (DTCs) framework. The research reported in this paper describes the approach to create the framework. This is followed by a demonstration of the framework as a basis to highlight characteristics of design tools considered important by designers at different stages of the industrial design process. Hence, the uses of the tools can be discussed in more systematic ways. We anticipate the framework can also become the basis for the more rigorous quantitative rating of characteristics highlighted, which will be the subject of future study.

\section{UNDERSTANDING DESIGN TOOLS}

Designers use design tools to externalise design ideas from their brains to our real world (Self, 2011). Designers nowadays use an expanding inventory of design tools during their design practice (Pipes, 2007; Self, 2011) due to emerging technology. In spite of the significant benefits, the selection of tools during the design process becomes more challenging for designers. Therefore, the evaluation of various design tools is vital for the utilisation of design resources. Design tool characteristics can be used to describe a design tool by breaking down its entire property to analysable and comparable factors (Self, 2011; Stolterman et al., 2009; Purcell and Gero, 1998). To understand and analyse design tools, some researchers built frameworks of some design tool characteristics. In this section, two key frameworks which share similar research purpose and design field with this study are reviewed.

\subsection{Self's Five Universal Tool Characteristics (UTCs)}

Self et al. (2009) propose a framework named the Five Universal Tool Characteristics which describes five common characteristics to investigate when analysing the capabilities of design tools in supporting designers to conduct design activities. The five universal characteristics are termed:

- Mode of Communication: This characteristic describes to what extent the design tool supports communication of design ideas with others and the designer's self.

- Level of Ambiguity: The extent of ambiguity that a design tool can support.

- Transformational Ability: The transformations of thinking occurring in design can be categorised into lateral and vertical transformations. The lateral transformation is the movement from one idea to a new idea while the vertical transformations refer to developing variations of the same idea.

- Level of Detail: The extent of detail as well as the amount and display of information in visual representations.

- Level of Commitment: It describes that design ideas that are created with certain design tools may be perceived closer to or further away from completion by stakeholders in terms of requirements for manufacture. 


\subsection{Purcell and Gero's five characteristics of sketching}

Focusing on one of the most commonly used design tools-sketching, Purcell and Gero (1998) summarise five inter-related characteristics of sketching.

- Reinterpretation: The emergence of new ways to interpret existing representations into design ideas or "Moves" between representations and new ideas (Schon and Wiggins, 1992).

- Denseness and Ambiguity: A similar concept as Ambiguity of Self's framework. Similarly, Purcell and Gero (1998) emphasis that the ambiguity characteristic of sketching results in reinterpretation.

- New Knowledge/Information: The perception/generation of new perceptual/conceptual knowledge become part of the problem-solving process when using sketching.

- Cyclic Process: The movements from reinterpretation and generation of new knowledge to further reinterpretation addressed and access to new knowledge during the use of sketching.

- Level of Expertise Related: The Level of Expertise influences designers, not only in terms of the use of tools but also their cognitive activities.

\subsection{Design tools and designers}

Self et al. (2009) and Purcell and Gero (1998) indicate a similar fundamental way of understanding and analysing design tools as well as designers/design activities which illustrate the feasibility of using frameworks of design tool characteristics to evaluate design tools. It's worth noting that the result of Self's Five Universal Tool Characteristics (UTCs) is very general while Purcell and Gero (1998)'s framework is only specific to (traditional) sketching. According to a survey of the literature, there is scope to add more characteristics of design tools to a more comprehensive framework which generalises across various design tools in industrial design.

The two frameworks indicate two perspectives of investigating design tools, namely the tool's capabilities and those of the users. The UTCs framework concludes some broadly shared characteristics of design tools for revealing and analysing designers' attitudes towards the tools (Self, 2011, 112). In other words, it is built from the perspective of design tools' properties/capabilities and their relationship with designers. Differently, the five characteristics of sketching are identified regarding the use of sketching(Purcell and Gero, 1998) and it reveals another important perspective to analysis design tools: the designers. As the Level of Expertise Related mentioned above, the effects on tools from designers are necessary and significant to consider. Therefore, a more comprehensive framework for design tool evaluation should reconcile both the designers and the design tools themselves. Next, building on these two perspectives, more characteristics of design tools are identified from a survey of literature so a more comprehensive framework for understanding design tools and conducting comparisons, namely the Design Tool Characteristics (DTCs) framework, is built to help optimise and select which tools to use and when during the industrial design process.

\section{CREATION OF THE DESIGN TOOL CHARACTERISTICS FRAMEWORK}

A more comprehensive framework of Design Tool Characteristics (DTCs) which reconciled both the perspectives from the tools and the designers is now built for understanding design tools to optimise the use of design resources in industrial design.

\subsection{Method}

As in Figure 1, the Capability-related Characteristics of Design Tools (CCs) are identified from the perspective of capabilities that designers get from using the tools. The User-related Characteristics of Design Tools (UCs) are identified from the perspective of designer's physical/mental input to the use of tools. Furthermore, it is contended that the interaction between the designers and the use of design tools can form an iterative cyclic process. Due to this interactive and inseparable relationship between designers and design tools, this adapted framework for design tool evaluation includes both the CCs and UCs. According to the literature, there are more characteristics could be taken into account. Some studies on design tool performance mentioned many "less obvious" characteristics of design tools. By looking for commonality and overlap characteristics in these studies, more comparable characteristics are next drawn into the proposed DTCs framework. 


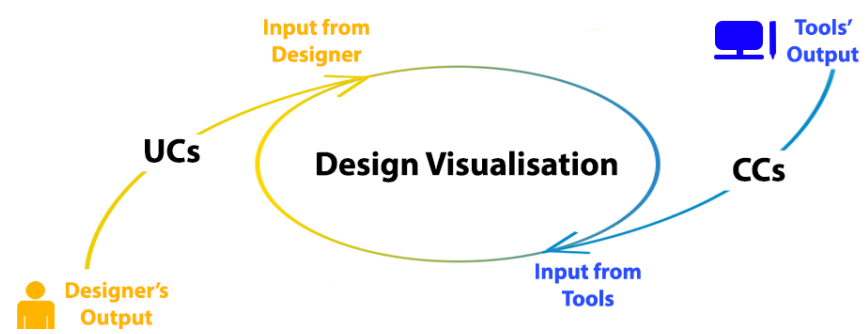

Figure 1. The interaction between designer and the use of design tools (Author's Own)

\subsection{The Capability-related Characteristics of Design Tools (CCs)}

As with the UTCs framework (Self et al., 2009), characteristics listed here are identified from the perspective of tools' capabilities which could help to reveal the potential of design tools regarding their capabilities in supporting designers. Different from the characteristics that identified from the perspective of the tool users (designers), the focus of the Capability-related Characteristics of Design Tools (CCs) is on the intrinsic properties of both the design tools and the representations that created with the tools.

- Ambiguity: The characteristic refers to "the extent of ambiguous and unambiguous representation of ideas" (Self et al., 2009) that tools can support. It is the vagueness makes multiple interpretations to happen. As mentioned in published researches (Stones and Cassidy, 2010; Goldschmidt, 1991; Alcaide-Marzal et al., 2013; Booth et al., 2016), the level of ambiguity in design representations, which also represents the level of abstraction, could affect the effectiveness of design ideation/conceptualisation process (Cross, 1999).

- Internal Communication: By breaking down the Mode of Communication of UTCs framework (Self et al., 2009), this framework has Internal Communication and External Communication to more precisely analyse design tools. The Internal Communication activity could be referred to the "Dialogue with Self" (Goldschmidt, 1991). This characteristic means the capability of design tools on supporting the self-reflective activity during the design process.

- External Communication: The modes of communication includes communicating design ideas via self-reflection to oneself (Lawson, 2006, Goldschmidt, 1991) as well as communicating design intentions via design embodiment to others(Self, 2011). This characteristic could be seen as the extent of support the designers can get from design tools/representations when communicating intentions to stakeholders/teammates.

- Problem Re-framing: Problem Re-framing happens when the use of certain design tools provides/supports an environment in which previous problem frames can re-emerge, be re-evaluated, combined and revised (Self and Pei, 2014). Differ from Internal Communication that focuses on the solutions; Problem Re-framing means reconstructing the understanding of the design problems. This characteristic describes the extent of support on Problem Re-framing activity that designers can obtain from the tools.

- Lateral Transformation: Considering the differences of tools on supporting different types of transformations, the Transformation Ability (Self, 2011) has been broken down into two characteristics. The Lateral Transformation represents the breadth of ideas, the "generation of new ideas" (Alcaide-Marzal et al., 2013).

- Vertical Transformation: Vertical Transformation is the variation of the same idea when the Lateral Transformation is not sufficiently supported. Also, it is known as detail development or "Depth-first transformations" (Cross, 2001), of which the occurrence frequency usually goes up with the progress of the design process.

- Level of Commitment: Same as the Level of Commitment in the UTCs framework. Since different types of representations may indicate different Levels of Commitment, this characteristic could be misleading and unwanted if tools are used at less appropriate phases (Pipes, 2007; Self, 2011).

- Fidelity: The Fidelity here can be understood as the accuracy of communicating the inner Ideas or the resemblance of target concept "Externalisation" (Self and Pei, 2014; Stones and Cassidy, 2010).

- Accuracy: This characteristic represents how precise the mechanical/ dimensional information is supported by the design representations/tools. 
- Mobility: Mobility or "Portability" (Evans and Aldoy, 2016) could refer to the physical mobility of both the design tool and the representation format that created with the tool. It relates to the conveniences of using the design tools.

- Immediacy: The delay between the creative inspiration and digital representation has been noted by some researchers (Aldoy, 2011; Evans and Aldoy, 2016). The characteristic identified is termed as Immediacy in this study, and it refers to the fluidity/continuity of doing-displaying process when designers are using the design tools. It could help to reveal the relationship between creativity flow and the fluency/accuracy of visualisation feedback.

- Flexibility: This refers to the freedom of changing/developing ideas with design tools. It is related to many aspects of the design tool, such as the device material, the interface, capability to undo/erase/dispose former representations (Buxton, 2010) and the number of steps to make changes or add details (Ranscombe et al., 2017). It could be related to the "Sunk Cost" (Viswanathan and Linsey, 2011), and the higher the "Sunk Cost" is, the unlikely the development/change of ideas would happen.

- Level of Details: Same as proposed by Self et al. (2009). In addition, Chen (2007) adds that it also refers to the level of engineering and manufacturing information as well as the level of form detail-complexity represented.

- Level of Aesthetics: This characteristic here refers to the general attractiveness of representations that created with the design tools, and it could be related to the style/type/amount of the renderings. The Level of Aesthetics could influence designers' way of thinking and doing. For instance, the higher level of aesthetics that a design representation has, the higher possibility that a design idea will survive due to its visual attractiveness. Self (2011) also notes the difference between this and the Level of Ambiguity that the Level of Detail "describes the embodiment of more or less specific information" while the Level of Ambiguity is used to denote opaqueness of ideas.

- Amount of Representations: Buxton $(2010,111)$ states that sketches are usually plentiful and the renderings of ideas tend to exist in the context of a collection which could potentially stimulate design creativity. Thus, the Amount of Representations and the way the representations are distributed(isolated or in a collection) could also be a significant DTCs that needs to be considered.

- Holistic View of Objects: Seeing design ideas in context can assist designers to think more clearly. Hence, if designers could thoroughly view the current design objects/representations through the tools used, the more comprehensive the designs could potentially be. Also, this characteristic may relate to the scalability/size/material of the tool/interface and the loading time of the views etc.

- Compatibility: Compatibility here refers to the capability of the design tools to ease the transitions in a design process, including the transition of media from traditional to digital visualisations, the transition from $2 \mathrm{D}$ to $3 \mathrm{D}$, and the transition from digital visualisation to physical prototype/production.

- Use Cost: According to literature, the Use Cost can relate to the average time invested and cognitive demand of creating a self-satisfied or client-satisfied representation. Equally, it could be divided into time and mental efforts consumed. As Bilda and Demirkan (2003) state that digital media could be time-consuming because designers have to deal with software attributes during design. At the same time, the "Sunk Cost" occurred during the design process can be lowered by the use of digital media, e.g. the redo/undo features can save the effort of redrawing/remodelling/redesigning. According to the literature reviewed, the general Use Cost of many design tools hasn't been fully investigated or noted except Traditional Sketching. In addition, the specific Use Cost of a design tool during a design case could be varied due to designers' different expertise and different design phases. However, the necessity of generating and spreading the knowledge of the general Use Cost, which could improve the effectiveness and efficiency of the use of tools, is relatively obvious.

- Learning Cost: Logically, compared to the Use Cost, we noticed the Learning Cost from design practice. This characteristic similarly affects design as the Use Cost does. It refers to the general time invested and cognitive demand of learning a design tool. This characteristic could potentially affect the selections of tools during the design practice and the long-term loyalty of the tools. Supposedly, Learning Cost could be optimised by using different learning materials, the timing or sequence of learning of tools, teaching methods as well as some environment settings. Therefore, 
to thoroughly evaluate a design tool and to create an "Effective Learning Experience" for design students, the analysis and study should take the Learning Cost into account.

\subsection{User-related Characteristics of Design Tools (UCs)}

The User-related Characteristics of Design Tools (UCs) here refer to the characteristics related to physi$\mathrm{cal} / \mathrm{mental}$ input of the users/designers that have influences on the use of design tools, are identified and termed:

- Tendency to Switch Tools: This characteristic describes the designer's tendency of switching tools, which could be related to specific real-time design requirements as well as be interlinked with other CCs of the tools. This tendency can result in personal preference of specific tools, and sometimes the switching activity could also help stimulate creativity (Shih et al., 2015). However, it is noteworthy that the activity of switching tools could also have some kind of negative influences on design creativity due to the time/mental/financial costs.

- Expectation: The Expectation of the outcomes from using a specific tool can influence when and how the tool is used during the design process. For instance, the Expectation of a high-resolution visualisation from using digital design tools may cause immersive use of rendering features and be time-consuming.

- Emotional Commitment to Ideas: This refers to the attachment to existing ideas and the reluctance to change ideas (Self, 2011; Viswanathan and Linsey, 2011). When it happens too early in a design process, it will lead to the unwelcomed premature "design fixation" (Crilly, 2015).

- Expertise: This characteristic means the level of experience/skills/knowledge that designers already have which could change the way and the outcome of using design tools. Various study results of the relationship between the "expertise" of designers and the performance of designers (Booth et al., 2016; Crilly, 2015; Robertson and Radcliffe, 2009) indicate that it is necessary to consider the effects from the Expertise of designers when evaluating design tools.

- User Accessibility: This characteristic means the general accessibility of design tools for design students/designers, which is potentially related the purchase/maintenance costs as well as the User Share in the market and industry. Also, the performance of designers is likely to be affected by this characteristic so the User Accessibility of tools should be taken into consideration when evaluating design tools.

- User Loyalty: This concept is derived from the "Customer Loyalty" and observed from design practices, it refers to the attitudinal and behavioural tendency of designers to favour a design tool. It can influence the selection and use of tools. As an illustration, a heavy/extreme loyal user of one certain tool might feel and suffer more difficulties when being engaged in other tools.

- User Share: This concept is derived from the "Market Share" in business fields, which means the percentage of users that certain product has in the local market. Hence, the design tools' User Share could be the percentage of users that they own in the industry, education and even in different design phases. This characteristic may have subtle effects on the designer's minds, and it also indicates the tools' capability from the perspective of a user's practical selection. Besides, the User Share of the industry can influence the selection of design tools in individuals and education. For example, if the local industry has strong manufacturing sector and high User Share on related design tools, the local design institutions/designers may focus more on those design tools.

\subsection{Considerations in the creation of the Design Tool Characteristics (DTCs) framework}

The framework of the Design Tool Characteristics (DTCs) is presented in Table 1. It combines the perspectives from both the design tools and designers, and it also covers more comparable characteristics, which offers the foundation to understand design tools at different stages of the industrial design process. Prior to testing/demonstrating the framework, we anticipate some grey area between the CCs and the UCs. For example, the Use Cost and Learning Cost are categorised as CCs at this stage, yet they are also related to the user's expertise to a certain degree. They are categorised as CCs because the intrinsic properties of a tool are more likely to have more influence on the Use Cost. Drawing a sphere on paper costs less time than turning on a laptop then creating an equivalent representation in a 3D modelling software due to the number of steps involved. We also expect some characteristics in the framework 
will be interlinked, which may cause some difficulties when distinguishing them in the application of the framework. Finally, it is also possible that the DTCs of a tool may vary depending on the context used. For example, the Level of Aesthetics of hand sketches may be considered high when designers communicate with each other, but it may be considered lower when designers communicate with clients. To summarise, there are considerations regarding the framework and its application so validation of the

Table 1. Table of Capability-related Characteristics of Design Tools (CCs) and User-related Characteristics of Design Tools (UCs) (Author's Own)

\begin{tabular}{|ll|l|}
\hline \multicolumn{3}{|c|}{ Design Tool Characteristics (DTCs) } \\
\hline \multicolumn{2}{|c|}{ Capability-related Characteristics(CCs) } & User-related Characteristics(UCs) \\
\hline Ambiguity & Immediacy & Tendency to Switch Tools \\
Internal Communication & Flexibility & Emotional Commitment to Ideas \\
External Communication & Level of Details & Expectation \\
Problem Re-framing & Level of Aesthetics & User Accessibility \\
Lateral Transformation & Amount of Representations & Expertise \\
Vertical Transformation & Holistic View of Objects & User Loyalty \\
Level of Commitment & Compatibility & User Share \\
Fidelity & Learning Cost & \\
Accuracy & Use Cost & \\
Mobility & & \\
\hline
\end{tabular}

framework is required in future studies. At this stage, this paper focuses more on presenting the creation of the DTCs framework. A brief demonstration of its application is now given.

\section{DEMONSTRATION: UNDERSTANDING THE USE OF DIGITAL SKETCHING WITH THE DESIGN TOOL CHARACTERISTICS FRAMEWORK}

As a brief demonstration of the use of the Design Tool Characteristics (DTCs) framework, a case study of four designers in a local design firm regarding their use of digital sketching is conducted.

\subsection{Investigation of digital sketching in practice}

Digital Sketching has been used in design practice and aroused interests of research in academia (Alcaide-Marzal et al., 2013; Evans and Aldoy, 2016). A review of the related literature shows that Digital Sketching could be a very effective tool for industrial design. Meanwhile, the general knowledge about the characteristics of this tool is not sufficient for designers to maximise the benefits of using it. The local design team which is sufficiently equipped with advanced digital sketching tablets has relatively low usage of this tool in design practice. In this case study, we demonstrate the use of the framework to highlight characteristics that are important to designers when considering the use of the Digital Sketching in comparison with Traditional Sketching and CAD. Doing so will help to understand the reasons behind the limited use of Digital Sketching in industry.

\subsection{Data collection}

The observation participants are four industrial designers who are using different design tools in their daily work at a local design firm. In 2 weeks, 8 sessions of observation were taken at their workplace. Each observation session includes 25 minutes of non-interference video recording and a short followup interview reflecting their behaviours. To analyse the manifestation of design tools in real design practice, designers' use of tools and choice of projects were based on their work at the moment. As in Table 2, there are 4 sessions of using Traditional Sketching, 2 sessions of using Digital Sketching and 2 sessions of using 3D CAD modelling among the 8 sessions of observation. Data collected are analysed using the DTCs framework. Behaviours and comments from the designers are mapped to related DTCs

Table 2. Participants and design tools

\begin{tabular}{|l|l|l|}
\hline Participant 1 & Senior Designer (10 Years) & Traditional Sketching \\
\hline Participant 2 & Senior Designer (7 Years) & Digital Sketching \\
\hline Participant 3 & Junior Designer (2.5 Years) & CAD Modelling \\
\hline Participant 4 & Junior Designer (1.5 Years) & Traditional Sketching \\
\hline
\end{tabular}


then discussed, for example, "quickly", "longer time" are mapped to the Use Cost, "messy", "nice" are mapped to the Level of Aesthetics.

\section{RESULTS AND DISCUSSION}

\subsection{The use of DTCs framework}

In the demonstration, the use of the Design Tool Characteristics (DTCs) framework to understand the design tools is effective. Data collected from designers are mapped to the DTCs and thus can be grouped and compared. The framework helped to highlight the important DTCs for each tool in design practice based on verbal or behavioural data collected thus further discussions can be made. From the 26 DTCs, 7 DTCs are revealed as the key DTCs that influence the use of Digital Sketching in sketching based on the frequency mentioned or behaviour related in the observation sessions. The connections between these 7 key DTCs are discussed in Subsection 5.2. As a result, the reasons behind the limited use of Digital Sketching in the specific design firm are clarified, and the opportunity to use it more efficiently and effectively is also indicated. Since the framework at this stage is aiming to provide guidance for qualitative analysis, the judgement of whether Digital Sketching is better or worse based on numeric ratings is not relevant to the research aim.

As expected, some of the DTCs in the framework are highly interlinked, which challenged how the mapping method approaches. For example, Fidelity can influence the Use Cost and also has an impact on Internal Communication as discussed above. Similarly, designers' attitude towards the tools is conditional to the purpose of the use. Hence, a more clear approach to use the framework can be developed in future studies. To summarise, the DTCs framework offers an effective and systematic way to understand design tools in-depth.

\subsection{Key DTCs for applying digital sketching in design practice}

According to observation, a few design tool characteristics are identified as the key DTCs that can affect the selection and use of Digital Sketching in industrial design practice. Figure 2 illustrates that balancing the need for these key DTCs, namely Accuracy, Ambiguity, Level of Aesthetics, and Fidelity, can optimise the Use Cost of Digital Sketching for more efficient Internal and External Communication. Specifically, the expected Level of Aesthetics of the representations has an impact on the perceived Use

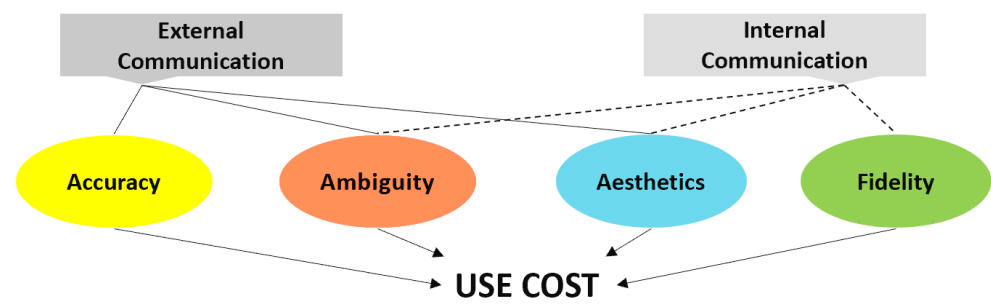

Figure 2. Key DTCs for optimising the use of digital sketching

Cost of the tool. For instance, the number of traditional sketches created in one observation session can vary from 2 to 18 due to the different level of Aesthetics. Whereas participant 2, an expert of Digital Sketching, can only optimise the shading of part of the design in one session using Digital Sketching to achieve a photorealistic sketch. The Use Cost of Digital Sketching increases more extremely when the level of Aesthetics increases. It may be partly because of a higher expectation of Aesthetics from digital sketches in general, and partly because of Digital Sketching makes it easier to achieve a higher level of Aesthetics with its the advanced features/control. If the designers adjust their expectation of Aesthetics on digital sketches for the right context, the Use Cost of Digital Sketching can also be lowered and thus encourage more efficient use of this tool.

Digital Sketching can remain a considerably high level of Ambiguity with low Use Cost if the tool is used in certain ways. For example, participant 2 demonstrated a few techniques of using Digital Sketching with high Ambiguity and Low Use Cost, namely "colour blocks", "silhouette" and "speed form sketching". Hence, Digital Sketching can be a powerful tool for ideation. However, the study also indicates some DTCs of Digital Sketching that can inhibit the designers to use Digital Sketching for highly ambiguous visualisation. As participants mentioned, "the tactile sensation is different", "(Digital 
Sketching) feels like it's sucking your energy" etc. The sensation difference in terms of behaviourcognition cooperation between pen/paper and digital tablets shows the Immediacy of Digital Sketching may cause mental stress of designers. Briefly, Digital Sketching can maintain high Ambiguity with considerable low Use Cost, but some other DTCs of Digital Sketching may offset this advantage and lead to its current limited use, e.g. low Immediacy and high Expectation of aesthetics etc.

Accuracy and Fidelity also have an impact on the Use Cost. For example, participant 3 built and tested one component of the design with CAD in a session while participant 1 using Traditional Sketching tested two solutions for the same project in one session. According to the participants, CAD modelling offers accurate dimensions but also requires high Fidelity-a clearer mental image-to start the work which leads to a higher Use Cost. Whereas Traditional Sketching is usually used to generate less accurate representations which also doesn't require high Fidelity, designers can start the work stress-free and be more "flexible" to change their ideas. However, traditional sketches might still be out of scale and require overlayed work later to get higher accuracy in CAD. This dilemma can be an opportunity to use Digital Sketching approaching Accuracy more efficiently. As digital sketches can be rescaled easily to test the design dimension with numerical inputs, participant 2 says that in one of his projects "since there are dimensional requirements, I did it in Photoshop (Digital Sketching) and made everything to scale in the beginning". Digital sketches can also remain at a lower level of Fidelity but with high Accuracy, so the Use Cost can remain low when ideating for mechanism/dimension solutions. All in all, Digital Sketching offers an intermedium range of Accuracy and Fidelity that can cover the gap between CAD and Traditional Sketching, balance the requirement of Accuracy and Use Cost, and remain low Fidelity for ideation.

\section{LIMITATIONS AND CONCLUSION}

This paper proposes a more comprehensive Design Tool Characteristics (DTCs) framework for understanding industrial design tools, and the creation of the framework is presented in detail. The DTCs framework describes the commonly shared characteristics among industrial design tools and offers a systematic way to understand design tools in the industrial design process. At this stage, considering the complexity of real-world design activities, the framework is built for guiding qualitative studies on understanding design tools. We anticipate the framework can also become the basis for the more rigorous quantitative rating of characteristics highlighted, which will be the subject of future study.

As a demonstration of its application, a case study on understanding the use of digital sketching in practice is conducted. Observation and follow-up interviews of four practising industrial designers in a local design firm were conducted using the framework. Analysing their behaviours and comments with DTCs framework illustrates the manifestation of Digital Sketching in industrial design practice. In terms of DTCs, Accuracy, Ambiguity, Level of Aesthetics, Flexibility, are highlighted as the key characteristics that influence the Use Cost of Digital Sketching when conducting Internal and External Communication. Thus our analysis suggests the balance between these four characteristics is vital for forming a better use of Digital Sketching in practice. Thus we show the framework helps us to understand which characteristics of the tool are most important to designers and establish a foundation on how to use and improve this tool in the future. A key limitation of the case study on understanding the use of Digital Sketching is used for demonstrating how the DTCs framework works. Hence, the investigation is not generalisable beyond the specific design firm.

It follows that the key limitation of the framework created is the need to further test its utility as a research tool. In the demonstration of its application, the coding of the DTCs in the framework evolved with iterations in intuitive coding. Equally, the different conditions and purposed of tool use become an important factor in design tool analysis. In future studies, more rigorous methods of applying the DTCs framework will be proposed with consideration of tool use context and purposes.

\section{REFERENCES}

Alcaide-Marzal, J., Diego-MÃąs, J. A., Asensio-Cuesta, S. and Piqueras-Fiszman, B. (2013), “An exploratory study on the use of digital sculpting in conceptual product design”, Design Studies, Vol. 34 No. 2, pp. $264-284$.

Aldoy, N. N. (2011), An investigation into a digital strategy for industrial design education, PhD thesis, Loughborough University. 
Bilda, Z. and Demirkan, H. (2003), “An insight on designers' sketching activities in traditional versus digital media", Design studies, Vol. 24 No. 1, pp. 27-50.

Booth, J. W., Taborda, E. A., Ramani, K. and Reid, T. (2016), "Interventions for teaching sketching skills and reducing inhibition for novice engineering designers", Design Studies, Vol. 43, pp. 1-23.

Buxton, B. (2010), Sketching user experiences: getting the design right and the right design, Morgan Kaufmann.

Chen, Z.-R. (2007), How to Improve Creativity: Can designers improve their design creativity by using conventional and digital media simultaneously?, Springer, pp. 571-583.

Crilly, N. (2015), "Fixation and creativity in concept development: The attitudes and practices of expert designers", Design Studies, Vol. 38, pp. 54-91.

Cross, N. (1999), "Natural intelligence in design”, Design studies, Vol. 20 No. 1, pp. 25-39.

Cross, N. (2001), "Designerly ways of knowing: Design discipline versus design science”, Design Issues, Vol. 17 No. 3, pp. 49-55.

Evans, M. and Aldoy, N. (2016), “Digital design sketching using the tablet pc”, Design Journal, Vol. 19 No. 5 , pp. 763-787.

Goldschmidt, G. (1991), “The dialectics of sketching”, Creativity Research Journal, Vol. 4 No. 2, pp. $123-143$.

Lutters, E., van Houten, F. J. A. M., Bernard, A., Mermoz, E. and Schutte, C. S. L. (2014), "Tools and techniques for product design", CIRP Annals - Manufacturing Technology, Vol. 63 No. 2, pp. 607-630.

Pei, E., Campbell, I. and Evans, M. (2011), "A taxonomic classification of visual design representations used by industrial designers and engineering designers", The Design Journal, Vol. 14 No. 1, pp. 64-91.

Pipes, A. (2007), Drawing for designers, Laurence King Publishing.

Purcell, A. and Gero, J. S. (1998), "Drawings and the design process: A review of protocol studies in design and other disciplines and related research in cognitive psychology”, Design studies, Vol. 19 No. 4, pp. 389-430.

Ranscombe, C., Bissett-Johnson, K., Boa, D. and Hicks, B. (2017), Designing with lego: Exploring the influence of low fidelity visualisation on collaborative design activities, in " $D S$ 87-8 Proceedings of the 21st International Conference on Engineering Design (ICED 17) Vol 8: Human Behaviour in Design, Vancouver, Canada, 21-25.08. 2017”.

Robertson, B. F. and Radcliffe, D. F. (2009), "Impact of cad tools on creative problem solving in engineering design", Computer-Aided Design, Vol. 41 No. 3, pp. 136-146.

Schon, D. A. and Wiggins, G. (1992), "Kinds of seeing and their functions in designing", Design studies, Vol. 13 No. 2, pp. 135-156.

Self, J. A. (2011), The use of design tools in industrial design practice, PhD thesis, Kingston University.

Self, J., Dalke, H. and Evans, M. (2009), "Industrial design tools and design practice: An approach for understanding relationships between design tools and practice", International Association of Societies of Design Research.

Self, J. and Pei, E. (2014), "Reflecting on design sketching: Implications for problem-framing and solution-focused conceptual ideation", Archives of Design Research, Vol. 27 No. 3, pp. 65-87.

Shih, Y. T., Sher, W. D. and Taylor, M. (2015), "Understanding creative design processes by integrating sketching and cad modelling design environments: A preliminary protocol result from architectural designers", 2015, Vol. 9 No. 3, pp. 17.

Stolterman, E., McAtee, J., Royer, D. and Thandapani, S. (2009), Designerly tools, in Proceeding Design Research Society Conference 2008.

Stones, C. and Cassidy, T. (2010), "Seeing and discovering: how do student designers reinterpret sketches and digital marks during graphic design ideation?", Design studies, Vol. 31 No. 5, pp. 439-460.

Viswanathan, V. and Linsey, J. (2011), "Design fixation in physical modeling: an investigation on the role of sunk cost", in ASME 2011 International Design Engineering Technical Conferences and Computers and Information in Engineering Conference, American Society of Mechanical Engineers, pp. 119-130. 